Revue des patrimoines

36 | 2018

Les archives photographiques de presse, pratiques comparées et enjeux méthodologiques

\title{
Copyright Doisneau / Rapho
}

Doisneau / Rapho Copyright

Interview de Francine Deroudille par Isabelle-Cécile Le Mée

\section{(2) OpenEdition \\ Journals}

Édition électronique

URL : http://journals.openedition.org/insitu/18194

DOI : 10.4000/insitu. 18194

ISSN : 1630-7305

Éditeur

Ministère de la culture

\section{Référence électronique}

Interview de Francine Deroudille par Isabelle-Cécile Le Mée, « Copyright Doisneau / Rapho », In Situ [En ligne], 36 | 2018, mis en ligne le 15 octobre 2018, consulté le 30 avril 2019. URL : http:// journals.openedition.org/insitu/18194; DOI : 10.4000/insitu.18194

Ce document a été généré automatiquement le 30 avril 2019

\section{(c) (i) (9)}

In Situ Revues des patrimoines est mis à disposition selon les termes de la licence Creative Commons Attribution - Pas d'Utilisation Commerciale - Pas de Modification 4.0 International. 


\title{
Copyright Doisneau / Rapho
}

\author{
Doisneau / Rapho Copyright
}

\author{
Interview de Francine Deroudille par Isabelle-Cécile Le Mée
}

1 Robert Doisneau (1912-1994) est l'homme d'une seule agence, Rapho, à laquelle il restera fidèle sa vie durant, des premières photos publiées juste avant la guerre aux dernières, réalisées peu avant sa disparition en 1994. L'histoire qui lie le photographe à son agence est professionnelle et photographique mais elle est surtout placée sous le signe de l'amitié avec Raymond Grosset d'abord, qui dirige l'agence de décembre 1945 à 1997, date à laquelle il passe le flambeau à ses enfants Mark et Kathleen avant de s'éteindre en 2000, mais aussi avec la communauté des photographes de l'agence : Ergy Landau (1896-1967), Ylla ${ }^{1}$ (1911-1555), Willy Ronis (1910-2009), Sabine Weiss (1924-) Édouard Boubat (1923-1999)...

2 Lorsque nous avons conçu le programme des journées d'études consacrées aux archives photographiques de presse, il nous a d'emblée paru essentiel d'étudier, à travers le parcours d'un photographe affidé à une agence, les mécanismes de production et de diffusion des reportages photographiques. Naturellement, nous avons pensé à ce couple emblématique formé par Robert Doisneau et l'agence Rapho. Nous avons donc sollicité Francine Deroudille, fille de Robert Doisneau, pour nous aider à comprendre cette singulière relation. Avec sa sœur Annette, Francine assure aujourd'hui la valorisation de l'œuvre de son père au sein de l'Atelier Robert Doisneau mais elle fut aussi, dès 1980 et pendant vingt ans, rédactrice à l'agence Rapho. Son regard et sa connaissance du fonctionnement de l'agence, de l'intérieur, sont autant de précieux atouts pour en appréhender l'organisation comme les principes de diffusion des tirages. Comme ayant droit, Francine Deroudille est aussi sollicitée sur les questions relatives au destin des tirages de Robert Doisneau dont certains sont aujourd'hui conservés dans des centres d'archives au sein de fonds de groupes ou de titres de presse. Cette double expérience rend son point de vue et son analyse sur cette question sensible intéressants et précieux.

3 L'entretien qui suit a été mené le 24 novembre 2015 lors des journées d'études organisées par les Archives nationales et la Mission de la photographie du ministère de la Culture. 
Isabelle Cécile Le Mée: Votre père commence très jeune à s'intéresser à la photographie. Après des études de graveur lithographe à l'école d'arts appliqués Estienne, il a 17 ans quand il entre, en octobre 1929, dans l'atelier de Léon Ullmann comme dessinateur de lettres ${ }^{2}$. Il y rencontre Lucien Chauffard qui dirige le studio photographique de l'atelier. Ce dernier va l'initier à la photographie avant de l'orienter vers André Vigneau qui, à l'automne 1931, cherchait un assistant ${ }^{3}$. C'est de cette période que datent ses premières parutions dans la presse comme ce reportage sur le marché aux puces de Saint-Ouen publié en 1932.

Francine Deroudille : Mon père est engagé par André Vigneau en $1931^{4}$. C'est pour lui une rencontre essentielle. Vigneau était un artiste dans l'atelier duquel passaient les grands artistes de l'époque : Dufy, Man Ray, les frères Prévert... Mon père découvre là un autre monde, celui de la création, des idées, de la vie intellectuelle. De plus, Vigneau était un homme généreux, noble. Imaginez, alors qu'il recevait dans son atelier Henri de Weindel (1868-1944), le patron du journal Excelsior, Vigneau propose à mon père, son petit opérateur de vingt ans, de montrer un reportage qu'il venait de faire en se promenant au marché aux puces (fig. 1). Le reportage a plu à de Weindel qui l'a publié dès la semaine suivante. Pour mon père, c'était un véritable coup de tonnerre. Lui, le petit assistant, publié dans un grand quotidien! C'était de l'ordre du rêve.

Figure 1

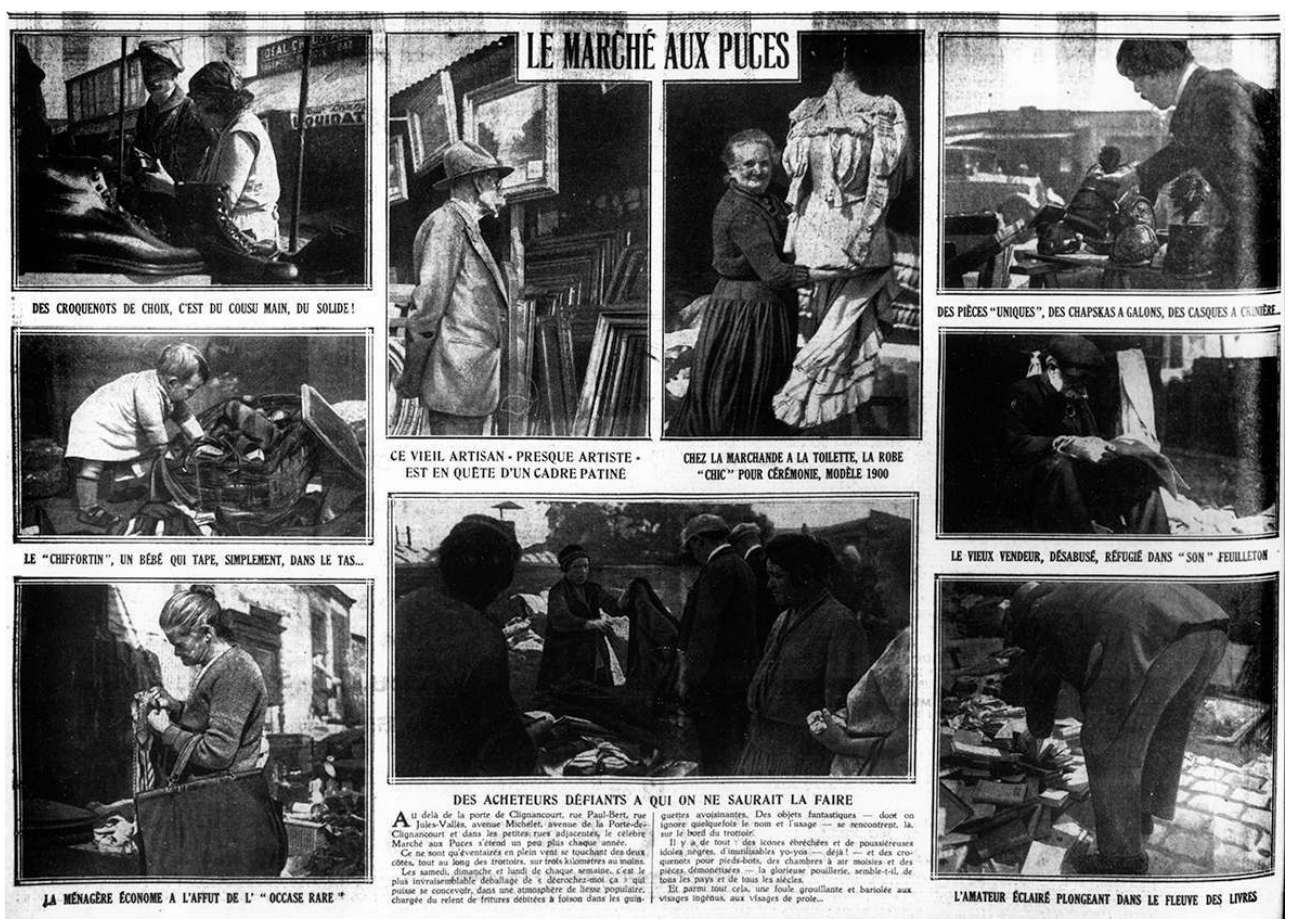

Le marché aux Puces, première parution d'un reportage de Robert Doisneau, I'Excelsior, 25 septembre 1932.

6 ICLM : En mai 1936, alors qu'il travaille comme photographe salarié chez Renault, plusieurs de ses photographies sont publiées dans le numéro 427 du magazine $\mathrm{Vu}^{5}$. On trouve, pour cette période, plusieurs de ses photographies dans la presse: comment procède-t-il alors pour trouver ses commanditaires et ses publications ? (fig. 2) 


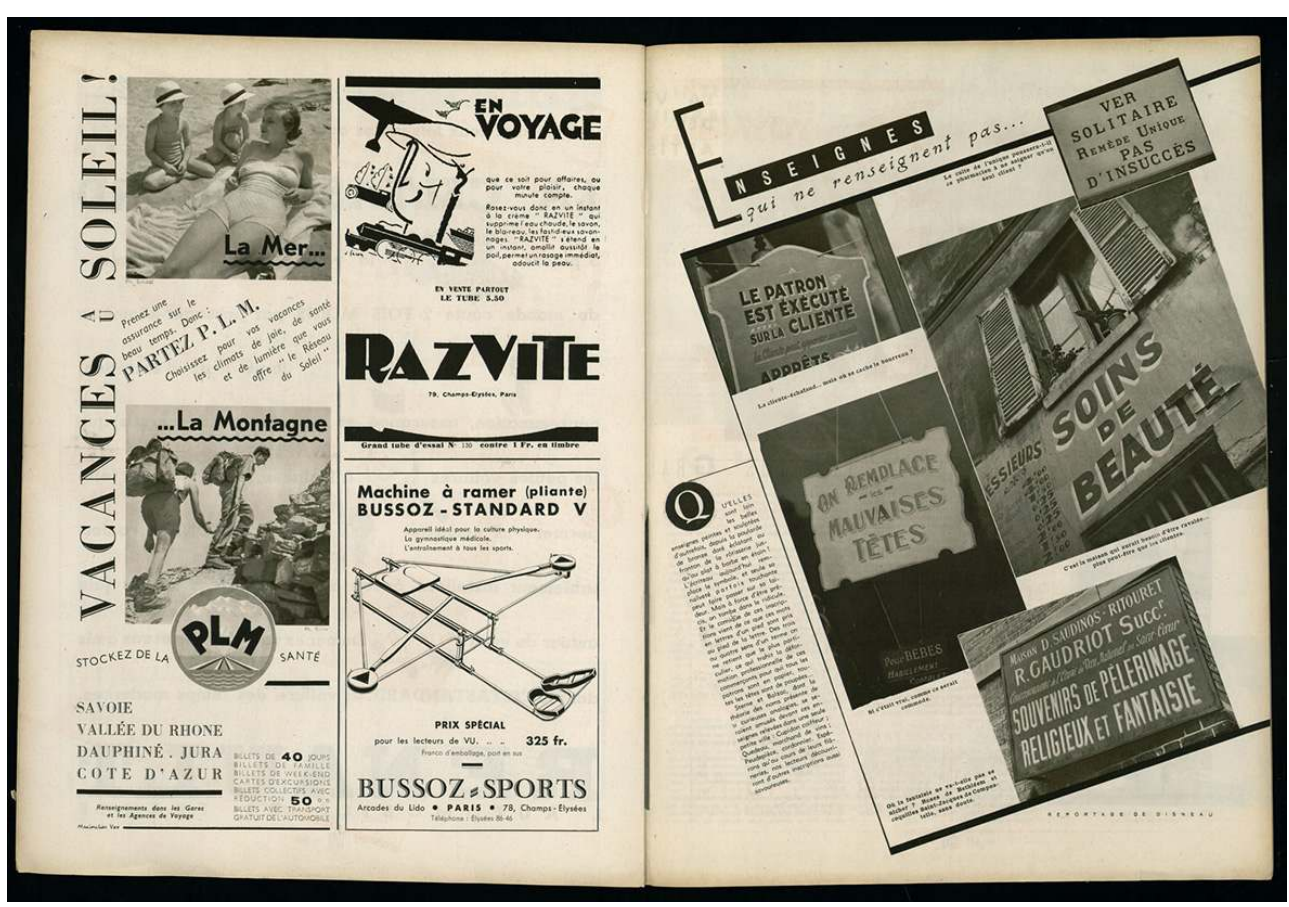

Enseignes qui ne renseignent pas, cinq photographies de Robert Doisneau, Vu, numéro 427, 20 mai 1936.

7 FD: Il ne publiait pas encore beaucoup avant-guerre mais le milieu de la presse photographique et des professionnels de la photographie était minuscule dans ces années-là. Les noms circulaient vite.

8 Le véritable essor de la presse photographique aura lieu dans les années d'après-guerre; c'est à ce moment-là que les commandes vont se multiplier. À la fin de sa vie, mon père s'amusait beaucoup à dire que ce qu'on voulait bien appeler "son œuvre" avait été réalisé avec du temps volé à ses employeurs. L'essentiel de son travail, surtout quand il est chez Rapho, était de répondre à des commandes. Tout ce qu'il a fait, ou presque, a été réalisé pour des commandes. Souvent, au cours de l'une d'entre elles ou en allant d'une commande à l'autre, il captait un instant, une situation, un visage et faisait une photographie qui «sortait des clous » et très souvent, c'était cette photographie-là qui était choisie, sélectionnée plus tard pour les publications.

9 Ce qui est certain, c'est que la rencontre avec Lucien Chauffard a été un fil conducteur extraordinaire pour mon père. De l'atelier Ullmann à Vigneau, de Vigneau à Renault, c'est Lucien Chauffard qui indique le chemin.

10 En 1939, c'est encore lui qui lui présente la photographe Ergy Landau, avec qui il a monté un studio Porte d'Ivry.

11 Ergy est hongroise, elle a été liée à László Moholy-Nagy, c'est une femme indépendante et brillante. La diffusion de ses photographies est assurée par un autre Hongrois, Charles Rado, qui vient de créer une petite agence de diffusion qu'il a appelée Rapho sur le conseil de son ami [le journaliste] Henri Calet. 
12 ICLM : Robert Doisneau se rapproche de l'agence Rapho en 1939. Qu'est-ce qui motive le rapprochement de votre père avec cette agence ? Un manque d'appétence pour la dimension administrative et commerciale du métier ?

13 FD : En 1939, mon père qui travaillait toujours aux usines Renault rêvait de devenir un photographe indépendant. Ergy Landau organise alors un premier rendez-vous entre mon père et Charles Rado. La première agence Rapho fondée en 1933 avait une tonalité très hongroise ${ }^{6}$. Charles Rado représentait essentiellement ses amis, des photographes hongrois : Ylla, Ergy Landau, Brassaï, Nora Dumas...

14 Charles Rado était une personnalité merveilleuse, un homme de grande culture, doté d'un sens de la communication exceptionnel, il avait déjà un important réseau qui lui assurait une certaine notoriété au sein de cette profession naissante des photographes de presse.

15 Devant Rado, mon père évoque son souhait de devenir photographe indépendant. Ce dernier l'encourage, lui propose de venir lui montrer régulièrement ses nouvelles photos mais en homme sage, lui déconseille de quitter son emploi en usant d'un argument imparable, le salaire gagné au service publicité de Renault est inégalable pour un photographe indépendant. Mon père est rentré chez lui déçu par cette première rencontre mais avec le projet de présenter à chaque occasion ses travaux à Charles Rado. Quelques mois plus tard, licencié par Renault pour retards répétés, ayant pu acquérir un matériel sommaire mais correct grâce à ses indemnités de licenciement, il retourne voir Charles Rado qui lui demande quels sont ses projets immédiats.

16 C'était l'été et mon père devait partir descendre la Dordogne en canoë avec une bande d'amis. Charles Rado, enchanté, lui dit que c'est un sujet qu'il peut vendre à la presse s'il le traite de façon très précise, en insistant sur les sites préhistoriques et les activités des riverains.

17 L'histoire, hélas! en décide autrement puisque c'est en photographiant les grottes des Eyzies en Dordogne que mon père apprend la déclaration de guerre. Il remonte donc à Paris, où il est mobilisé. « Ce fut la fin de la jeunesse » dira-t-il plus tard.

18 Il laisse quelques photos de la descente de la Dordogne en canoë à Charles Rado qui les diffusera juste avant de quitter la France pour les États-Unis. Juif hongrois, c'est à New York qu'il va désormais travailler en fondant là-bas l'agence Rapho Guillumette Pictures.

19 ICLM : Quelques années plus tard, au sortir de la guerre en janvier 1946, votre père retrouve le chemin de l'agence qui n'est alors plus dirigée par Charles Rado mais par Raymond Grosset...

FD : En 1944, mon père, qui avait vendu des photos de la Libération de Paris à la presse, fut invité à entrer à l'ADEP qui ressuscitait une agence des années 1930, Alliance Photo7 ${ }^{7}$ Il y rencontra Henri Cartier-Bresson, ce qui marqua le début d'une grande amitié.

21 Pourtant il n'en gardait pas un bon souvenir car si les publications se multipliaient, il ne touchait pas les rémunérations auxquelles il aurait pu s'attendre !

22 Il décida donc de cesser sa collaboration avec Alliance Photo mais resta en très bonnes relations avec Maria Eisner qui avait co-fondé cette agence.

23 En janvier 1946, Raymond Grosset, qui venait de rouvrir l'agence Rapho, prit contact avec mon père, lui proposant de reprendre la collaboration ébauchée avec Charles Rado en 1939. 
La même semaine, Maria Eisner, qui venait de fonder l'agence Magnum avec George Rodger, Robert Capa et Henri Cartier-Bresson, l'appela pour lui proposer de les rejoindre.

Incroyable hasard, mais mon père se sentait moralement engagé avec Raymond Grosset qui lui avait fait une excellente impression. Il choisit donc Rapho contre Magnum, qui lui ressemblait moins et dont la vocation internationale l'intimidait un peu.

Plus tard, il dira que malgré l'amitié forte et complice qui le liait à Henri Cartier-Bresson, il n'avait jamais regretté son choix. Souvent, Henri avait réitéré sa demande pour le faire venir à l'agence Magnum mais mon père se sentait chez lui à l'agence Rapho, où il avait une indépendance totale. Les photographes de Magnum avaient des débats intellectuels puissants parfois violents mon père n'était pas du tout dans ce jeu-là. Chez Magnum, les photographes travaillaient essentiellement à l'étranger, beaucoup de choses se passaient entre New York et Paris; mon père ne parlait pas de langues étrangères et préférait explorer des lieux plus proches et familiers. Pour toutes ces raisons, Rapho était une agence qui lui convenait parfaitement. Toute sa vie, il lui est resté fidèle, extrêmement lié à sa direction.

27 Raymond Grosset lui donna immédiatement un sujet pour la presse, sur un groupe de naturistes qui vivaient dans des arbres à Meulan. Tout petit sujet de proximité, amusant à réaliser, qui fut publié immédiatement. Les droits furent réglés avec la même diligence, ce qui acheva de sceller la confiance que mon père portait à Raymond. C'est ainsi que se mit en place une collaboration extrêmement fluide, complice et de plus en plus amicale.

ICLM : Robert Doisneau incarne l'esprit de l'agence Rapho, les deux semblent indissociables. Comment peut-on qualifier la relation de travail qui liait Robert Doisneau et l'agence Rapho?

Raymond et mon père avaient à peu près le même âge mais Raymond était un peu comme un grand frère pour lui, il y avait une fluidité de relations entre eux qui fait qu'ils n'ont signé un contrat que très tardivement. Plus tard, des contrats ont été établis entre les photographes et l'agence mais en 1946, je ne crois vraiment pas que l'on puisse retrouver l'ombre d'un contrat ${ }^{8}$.

Tout fonctionnait à la confiance. Mon père souhaitait être débarrassé de toute recherche commerciale, de tout souci comptable pour lesquels il n'avait ni goût ni talent, pour se consacrer entièrement au travail photographique.

La complicité avec Raymond Grosset était telle qu'en 1949, lorsque mon père a été engagé par Vogue, c'est Raymond Grosset qui a négocié son contrat d'exclusivité avec Condé Nast ! (fig. 3) 


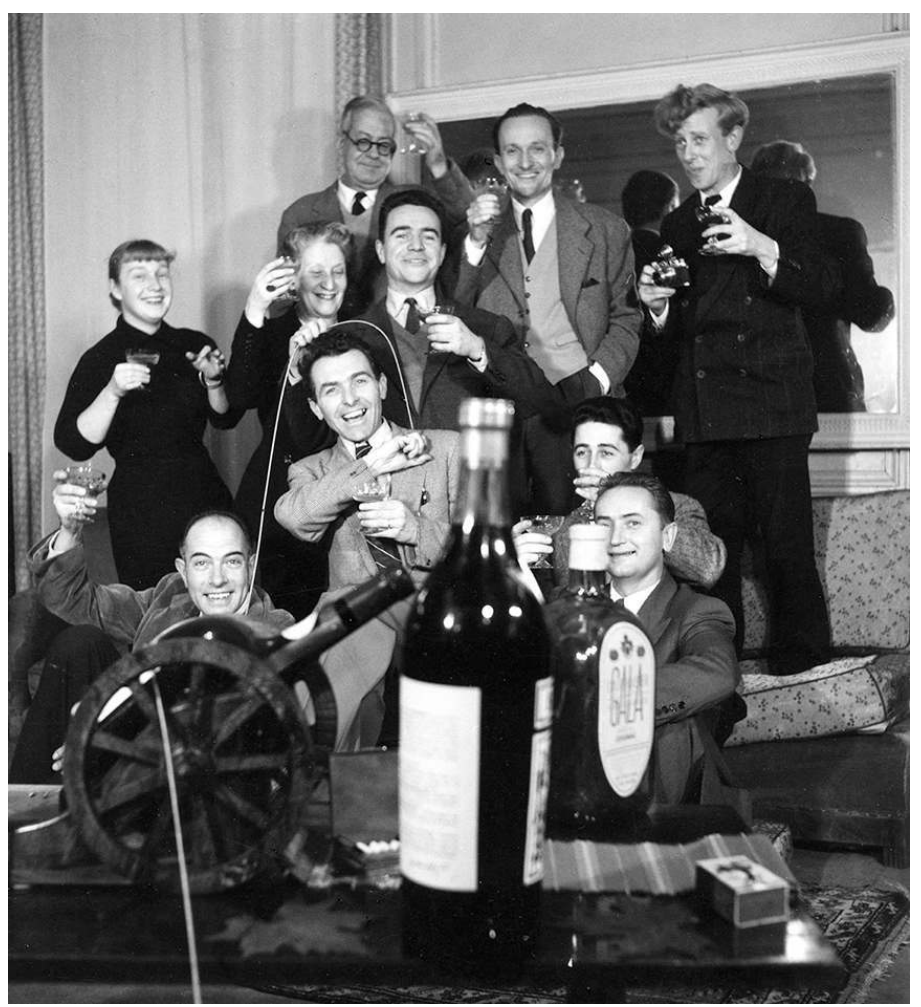

L'équipe de l'agence Rapho. Robert Doisneau (au centre avec le déclencheur à distance) entouré de Raymond Grosset, Eddy Van der Veen, Sabine Weiss, Emile Savitry, Ergy Landau, Serge de Sazo..., Noël 1953.

Phot. Robert Doisneau. ( ) Robert Doisneau / Rapho.

ICLM : Concrètement, quel rôle jouait l'agence pour les photographes qu'elle représentait ? À la fois dans la recherche de commandes, dans la production des sujets puis ensuite dans la recherche de publications et dans la diffusion des photographies?

FD : Complicité totale, mais là encore, je parle de l'histoire de mon père avec l'agence Rapho parce que, pour avoir travaillé à l'agence beaucoup plus tard, dans les années 1980, je sais que les relations avec l'agence pouvaient être différentes d'un photographe à l'autre.

Pour mon père, les commandes sont arrivées très vite ; en 1946 la presse photographique était en plein essor, les demandes étaient nombreuses, en particulier par la presse anglaise et américaine. Raymond travaillait beaucoup avec la presse américaine, avec le magazine Life particulièrement. Sans doute le fait que Charles Rado soit installé à New York n'était pas sans influence sur les nombreuses commandes passées par la presse américaine à Rapho. La photographie la plus célèbre de mon père, le Baiser de l'Hôtel de Ville, (son «Angélus de Millet» disait-il!) a été réalisée pour Life. L'agence recevait les commandes, les négociait et aidait les photographes à les réaliser. Le photographe restait propriétaire de son matériel mais déposait toujours une série de tirages à l'agence qui en assurait les reventes.

35 ICLM : Ainsi, au retour de son reportage, le photographe développait ses négatifs, tirait ses planches-contact et réalisait ses tirages. Le photographe réalisait-il lui- 
même son editing ou l'agence l'accompagnait-elle dans le choix d'une sélection d'images à diffuser? Qu'advenait-il des photographies non sélectionnées, des négatifs ? Étaient-ils conservés à l'agence ou les photographes s'occupaient-ils de gérer directement leurs archives? FD : Mon père faisait ses editings lui-même mais ce n'était pas le cas de tous les photographes. Un editing n'est pas le même si les photos sont destinées à la presse ou à l'édition, il arrivait donc que certains photographes confient leurs planches de contacts pour que les editings spécialisés soient réalisés à l'agence ou directement par le client. Chez Rapho, nous n'avons jamais voulu garder les négatifs, seulement des tirages, des tirages de travail $18 \times 24$. Peu de tirages plus grands, pour des problèmes de stockage. Quand mon père faisait un reportage ou même une simple prise de vue, il déposait toujours les tirages à l'agence. L'équipe de l'agence intégrait les nouvelles images dans le stock. Les photos étaient classées dans des boîtes thématiques, tous photographes confondus (fig. 4).

Figure 4

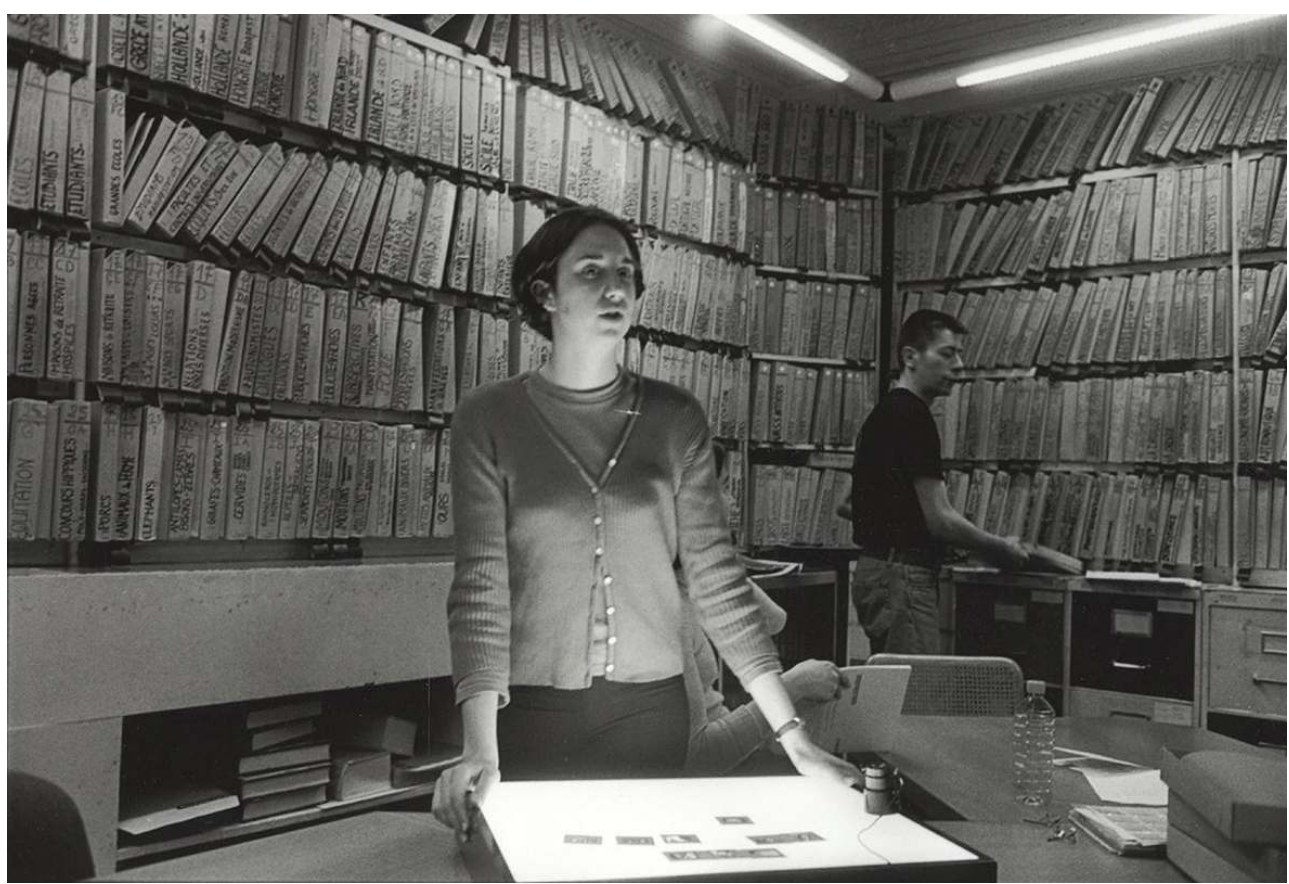

Dans les locaux de l'agence Rapho, années 1970

Phot. François Le Diascorn. (c) François Le Diascorn.

Il a fallu attendre les années 1980 pour que des boîtes d'auteurs soient réalisées. Doisneau, Boubat, Ronis, Charbonnier, Sabine Weiss étaient demandés pour leurs travaux personnels et non plus pour les sujets couverts mais cette identité d'auteur est tardive dans une agence comme Rapho qui se considérait comme une agence d'illustration photographique plus que comme une agence de photographes.

ICLM : Donc l'agence conservait pour la seule diffusion un jeu de tirages d'une infime partie du reportage produit, qui correspondait à l'editing réalisé par le photographe ou par un membre de l'agence? Ces tirages étaient prêtés à l'agence par le photographe pour la seule promotion et diffusion de son travail, ce dernier en restant propriétaire? 
41 FD : Absolument il s'agissait de tirages de consultation, et qu'on appelait à l'agence des tirages de travail. À l'agence Rapho, les photographes payaient les tirages, ils les déposaient donc à l'agence mais en étaient propriétaires. L'agence n'était propriétaire de rien mais agissait comme un intermédiaire entre le photographe, les commanditaires et les utilisateurs des images, et c'est à ce titre qu'elle percevait une commission.

42 Raymond voulait d'ailleurs aller très rapidement dans la restitution des tirages ou des diapositives aux photographes après que les editings aient été réalisés afin de ne garder que le "suc » de la production. Il évaluait très vite ce qui pouvait avoir un intérêt commercial pour l'agence. Il ne fallait surtout pas que les archives de l'agence s'encombrent de photographies supplémentaires, il y avait donc un va et vient constant des tirages entre l'agence et les photographes.

43 Rapho était une agence de presse photographique qui a gardé son statut d'agence de presse jusqu'à son rachat par l'agence Gamma. Néanmoins, la presse n'était pas la seule utilisatrice des photographies et une partie de l'agence était dédiée à la publicité, à l'édition, aux expositions. Au fil du temps, ces secteurs sont devenus de plus en plus importants.

44 Une agence de presse doit pouvoir justifier que l'essentiel de son chiffre d'affaires est réalisé avec la presse.

45 Pour que Rapho puisse garder le statut d'agence de presse et que ses employés conservent leur carte de presse, Raymond Grosset a racheté en 1979 l'agence TOP qui conservait et diffusait les archives d'Hachette-Réalités, principalement constituées des archives photographiques de la revue Réalités. L'agence Rapho facturait les publications presse et l'agence TOP le reste des utilisations : édition, expos, locations d'expos. C'est ainsi que Rapho a pu rester jusqu'à sa revente une agence de presse. au sein de l'agence Rapho ; en quoi consistait votre travail ? (fig. 5) 
Figure 5

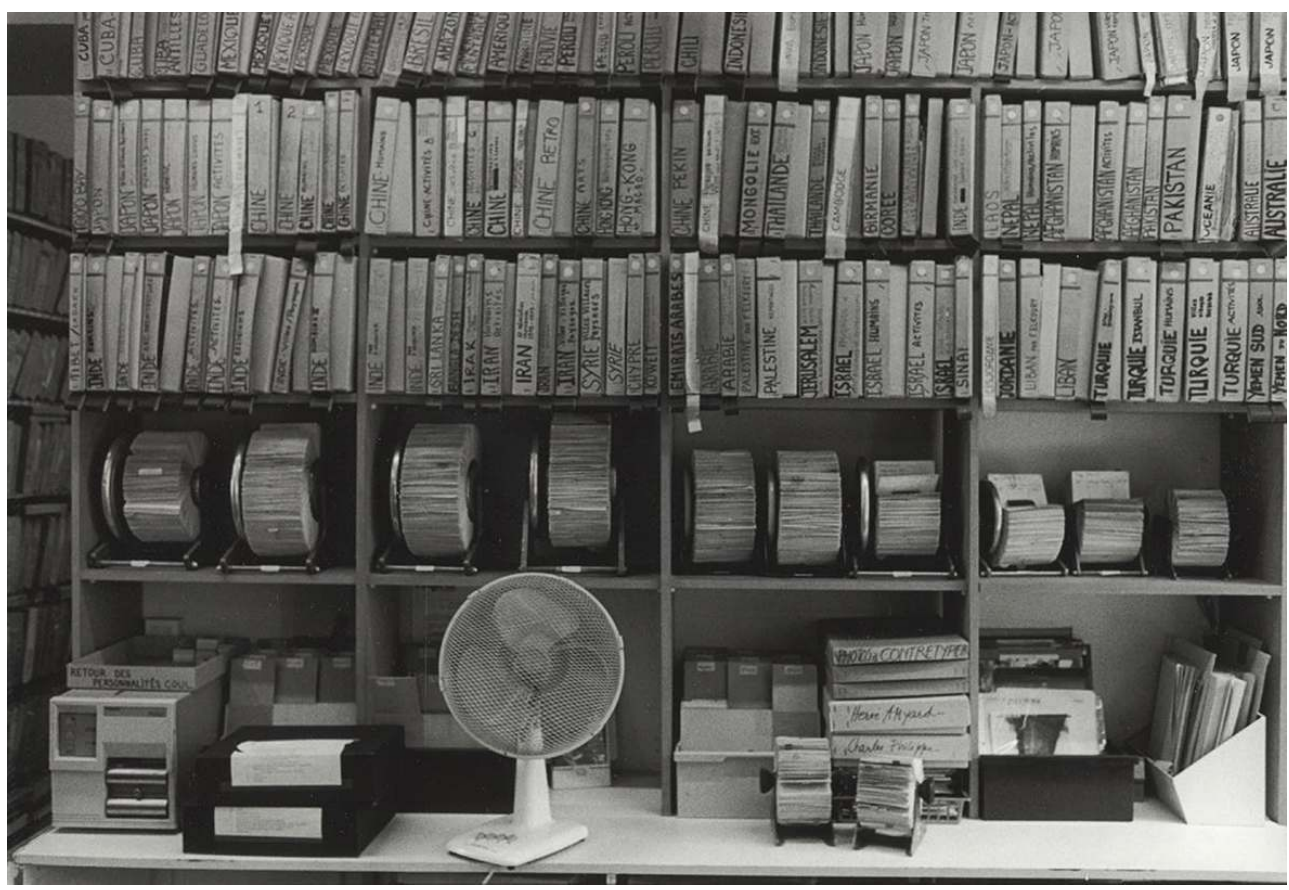

Agence Rapho, le classement des tirages en boîtes et instruments de recherche, années 1970.

Phot. François Le Diascorn. (c) François Le Diascorn.

FD : Nous étions très polyvalents au sein de l'équipe Rapho. J'y ai appris le métier étape par étape. Je me suis occupée un moment de la publicité puis de l'édition et des expositions qui ont vite été mes secteurs de prédilection. J'écrivais des textes d'accompagnement pour les sujets de certains photographes. Il y avait aussi un petit journal, Les Échos de Rapho, dont il fallait rédiger les textes. Mais il n'était pas question de rechigner devant la facturation, les reclassements, les recherches, les négociations. C'est une chose très précieuse pour moi maintenant d'avoir été formée à cette école-là. $\mathrm{En}$ contact direct avec la clientèle puisque les utilisateurs d'images venaient les chercher sur place à l'agence, et en connaissant tous les postes de la distribution d'une collection.

ICLM: Quel était le mode d'archivage des photographies au sein de l'agence? Comment la consultation s'organisait-elle?

FD: Une fois sélectionnées par le photographe puis par l'agence, les photographies étaient tamponnées avec le tampon de l'agence; jamais aucun tirage n'est sorti non tamponné de l'agence. Au verso des photographies, on peut retrouver des nombreuses annotations qui nous renseignent sur la vie des épreuves. Les caches des diapositives sont aussi couverts de renseignements : titre, date, boîte de classement. Les tirages (et les diapositives) étaient classés dans des boîtes thématiques. Noir et blanc d'un côté, couleur de l'autre (fig. 6). 


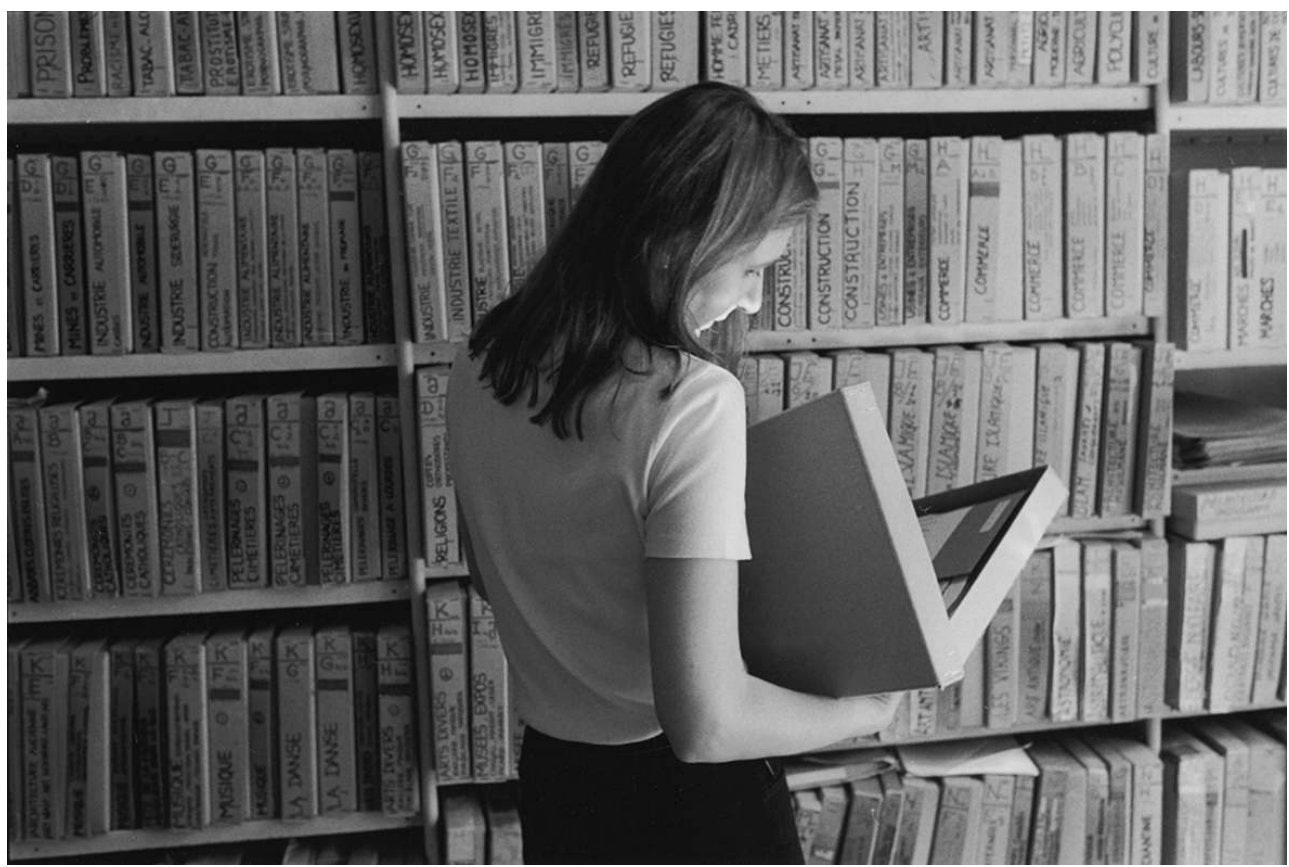

Dans les locaux de l'agence Rapho, années 1970.

Phot. François le Diascorn. (c) François Le Diascorn.

51 Ces boîtes servaient à la consultation et les tirages - ou les diapositives - qui intéressaient les clients, sortis de leurs boîtes, pour leur être confiés jusqu'à la réalisation de leur projet. Ensuite, les clients devaient les renvoyer à l'agence. Un service de relance existait d'ailleurs depuis 1948 pour réclamer le retour du matériel qui n'était pas effectué dans les temps.

52 Au moment de la sortie, un bon était établi, précisant la description de la photo, son utilisation prévue, la date de retour exigée et les conditions de vente.

53 Quand un client arrivait chez Rapho, la première chose que l'on faisait était de lui faire signer les conditions de vente en vigueur ; ces fameuses conditions de vente écrites en tout petits caractères que personne ne prend le temps de lire. Le nouveau client se trouve donc engagé à respecter les règles fixées par l'agence.

Une fois signées, ces conditions étaient classées dans le répertoire de la clientèle. À chaque fois qu'un tirage sortait, quelques-unes des conditions de vente qui nous paraissaient essentielles étaient rappelées sur le bon de sortie, comme par exemple le fait que la photographie était un prêt et qu'elle devait revenir dans les délais fixés lors de l'emprunt, ou que tout tirage non rendu serait facturé. Bref, des choses qui semblaient essentielles au bon fonctionnement de l'agence qui en effet avait besoin de récupérer ses tirages puisqu'ils constituaient son stock de diffusion.

Bien sûr, quand une photographie était très demandée, il existait plusieurs tirages de travail rangés dans cinq à dix boîtes différentes. Réalisés par les photographes ou sous leur contrôle, les tirages de travail étaient de beaux tirages barytés, qui bien sûr à l'usage perdaient de leur lustre mais tout de même, les tirages qui circulaient étaient des originaux de valeur. On retrouve parfois maintenant dans des ventes certains tirages 
tamponnés Rapho qui sont donc des tirages volés puisqu'ils auraient dû impérativement être rendus à l'agence après chaque emprunt. Quand quelqu'un posait pour lui, même s'il s'agissait d'inconnus photographiés dans la rue, mon père leur demandait une adresse et leur envoyait une épreuve. C'est donc normal que de nombreuses épreuves originales circulent. Mais ces tirages ne possédaient jamais le tampon de l'agence. Nous considérons, lorsqu'un tirage circule avec un tampon de l'agence, qu'il est volé. Un tirage qui n'a pas été rendu à l'agence est un tirage volé. Nous avons beaucoup de demandes d'expertises qui nous parviennent sur notre site Atelier Robert Doisneau et il faut souvent dire à nos interlocuteurs qu'ils sont en possession d'un tirage volé. Un tirage d'agence est une épreuve de travail et pas un tirage de collection, il n'a aucune raison de se trouver sur le marché, il doit donc être rendu à l'agence. Sur le site de la Fondation Henri Cartier-Bresson, Henri avait écrit un petit texte pour dire à quel point il ne souhaitait pas que ces tirages-là soient considérés comme des tirages de collection; avec le ton qui le caractérise, il explique que ce sont des tirages volés. Nous avons la même politique à l'Atelier Robert Doisneau, on ne demande pas aux gens de nous les rendre mais on leur demande d'en conserver un usage strictement privé, de ne pas les vendre car ils n'en sont pas vraiment les propriétaires.

ICLM : Des centres d'archives, des bibliothèques conservent des archives photographiques de presse provenant notamment de journaux ou groupes de presse. Parmi ces ensembles acquis ou déposés peuvent se trouver des photographies prises par Robert Doisneau diffusées par l'agence Rapho et non restituées comme l'exigeaient pourtant les conditions de cession. Quelle attitude adoptez-vous vis à vis de ces centres d'archives qui ne conservent plus des photothèques actives mais des fonds en provenance d'une entreprise de presse ?

FD : C'est bien que des photographies soient conservées dans les centres d'archives.

61 Nous avons réalisé en 2016 une exposition avec le Muséum d'histoire naturelle sur un reportage que mon père y avait effectué en 1942. À la suite de ce reportage qui avait été une commande de Maximilien Vox, un ensemble de tirages originaux était resté au Muséum où il était conservé. À partir de ces photographies, nous avons pu mettre au point une très belle exposition. Nous sommes ravies, Annette et moi, que cette collection soit conservée là par un personnel compétent qui réussit à la valoriser. Donc dans des cas analogues, quand un travail de conservation et de valorisation est réalisé, il faudrait vraiment être stupide pour en demander la restitution. Une convention est d'ailleurs signée avec le Muséum pour définir ce qui les engage.

62 À l'Atelier Robert Doisneau, nous souhaitons mettre en valeur l'œuvre, à travers la création d'ouvrages d'édition, d'expositions et ensuite leurs itinérances. L'essentiel de notre travail se situe là.

63 Nous considérons aussi qu'il est important de garder la collection dans son intégrité, nous ne vendons pas d'originaux, à l'exception des tirages signés qui étaient destinés dès leur origine à la vente aux collectionneurs. 

prenait conscience de leur intérêt, rare, précieux parce que minuscule et presque invisible au premier coup d'œil. Et maintenant, il nous arrive de ne pas tenir compte de invisible au premier coup d'œil. Et maintenant, il nous arrive de ne pas tenir compte de
recadrages et d'autoriser la publication de photos plein cadre. C'est une façon de lui désobéir en étant fidèles à sa façon de voir...

ICLM : Si l'on évoque la question relative aux droits d'auteur et particulièrement, au droit moral du photographe, votre père travaillait dans un format $6 \times 6$; en consultant la presse ou les ouvrages dans lesquels ses photographies sont publiées, il n'est pas rare de trouver des recadrages très différents d'une même prise de vue. Quelle était la position de Robert Doisneau sur ces nouvelles images?

FD : Mon père avait une formation de graphiste : il avait été formé quatre ans à l'école Estienne, il avait été dessinateur de lettres, il adorait préparer des petites maquettes pour ses reportages, pour ses projets de livres, la scénographie de ses expositions.

Il regardait ses photographies avec un œil de graphiste. Il a beaucoup travaillé au 6 x 6 puisqu'il utilisait le Rolleiflex. À partir de 1952, il a adopté également le 24 x 36 Leica puis Pentax ou Nikon. Il disait toujours que pour lui, une image faite au Rolleiflex était faite pour être recadrée. Ses planches-contact portent souvent des indications de recadrage. Il peut indiquer pour la même photo un recadrage en largeur et un en hauteur.

Prenons par exemple cette photographie qui a été beaucoup diffusée et qui s'appelle L'Information scolaire. Cette photographie a été prise en 1956 et elle montre un élève en classe, en train de copier sur un camarade. Cette photographie a souvent été utilisée pour faire des couvertures et donc elle a souvent été utilisée en hauteur et mon père l'a toujours recadrée en hauteur mais lui-même, quand il l'a publiée dans un livre, a choisi un cadrage en largeur et donc il a recadré en largeur avec un gros plan sur le regard de l'enfant qui copie.

8 C'est la même chose pour le Baiser de l'Hôtel de Ville : il a deux ou trois cadrages différents qui paraissent parfaitement harmonieux par rapport à la reproduction. Il s'adaptait à l'utilisation de l'image. Tout le contraire du filet noir d'Henri Cartier-Bresson.

Aujourd'hui on respecte sa volonté et on apprécie chaque demande au cas par cas. À la fin de sa vie, alors que nous faisions des sélections pour un ouvrage d'édition, il m'avait dit que plus le temps passait et moins il avait envie de recadrer les $6 \times 6$ car on trouvait toutes sortes d'éléments intéressants dans l'entourage de ce qui était l'élément principal de la photo, son centre.

Dans un premier temps, ces éléments lui avaient semblé des scories et le temps passant, il Propos recueillis par Isabelle-Cécile Le Mée

\section{NOTES}

1. - De son vrai nom Kamilla Koffler.

2. - L'atelier que dirigeait Léon Ullmann s'était spécialisé dans la publicité à destination de l'industrie pharmaceutique. 
3. - HAMILTON, Peter. Robert Doisneau. La vie d'un photographe. Préface Sabine Azéma. Paris: Hoëbeke, 1995.

4. - André Vigneau (1892-1968) est un artiste accompli. D'abord peintre formé à l'école des Beaux-Arts de Bordeaux, André Vigneau aborde tour à tour le cinéma, la musique, la décoration, la sculpture ou la photographie. Son fonds photographique est entré à la Bibliothèque historique de la Ville de Paris grâce à une donation de ses héritiers en 1984. Voir sur le site : https:// ccfr.bnf.fr/portailccfr/jsp/index_view_direct_anonymous.jsp?record=rnbcd_fonds:FONDS:4147 [consulté le 30/08/2018].

5. - Dans ce numéro daté du 20 mai 1936, cinq photographies composent une amusante page consacrée aux Enseignes qui ne renseignent pas. Le reportage est crédité "Disneau » et non Doisneau.

6. - Charles Rado (1899-1970), né en Hongrie en 1899, avait commencé à travailler à Berlin pour une maison d'édition avant de fuir l'Allemagne et la montée du nazisme pour rejoindre Paris où il fonde en 1933 l'agence Rapho (contraction de Rado Photo). En 2010, Michel Puech reproduit dans son blog un texte autobiographique de Raymond Grosset. Celui qui ré-ouvrira l'agence après la guerre y évoque ainsi Charles Rado : «C'est lui qui, avec sa concurrente Maria Eisner, a lancé ce genre d'agences travaillant à la commission et non plus employant des photographes salariés. » Il précise que ce lien nouveau assurerait au photographe « une indépendance identique à celle d'un peintre vis-à-vis d'une galerie assurant la représentation ...». Voir sur le site: https:// blogs.mediapart.fr/michel-puech/blog/290310/l-agence-rapho-racontee-par-raymond-grosset-lami-des-photographes [consulté le 30/08/2018].

7. - L'histoire de l'agence Alliance Photo commence en 1934 à Paris, au numéro 22 de la rue Vernier, où René Zuber possède un studio photographique. Autour de lui, quelques jeunes photographes donnent vie à ce que l'on appellera le Studio Zuber. Ils se nomment entre autres Pierre Boucher, Denise Bellon, Pierre Verger ou encore Émeric Feher. Dans le catalogue de l'exposition qu'a consacrée la Mairie de Paris à Alliance Photo en octobre 1988, Thomas Michael Gunther rapporte des propos de Pierre Boucher qui lui précise que Maria Eisner était passée au studio de René Zuber et avait ainsi fait leur connaissance au début de l'année 1934. Maria Eisner propose alors aux deux photographes de les représenter. Ce qu'elle fit avec brio. Alliance Photo est née. L'agence poursuivit son activité jusqu'en 1940. Maria Eisner est internée dans un camp dans le sud de la France avant de fuir l'Europe pour se réfugier aux États-Unis où elle co-fonde l'agence Magnum en 1947. Après la guerre, Suzanne Laroche, photographe et femme de Pierre Boucher, relancera avec quelques photographes l'activité d'Alliance Photo, renommée alors ADEP. GUNTHER, Thomas Michael, THÉZY, Marie de. Alliance Photo, agence photographique 1934-1940. Cat. exp, Bibliothèque historique de la Ville de Paris, 1988-1989. Paris : BHVP, 1988.

8. - Dans le documentaire Rapho, une agence parisienne, André Gamet, photographe, évoque ses premières années de collaboration avec l'agence, dès 1946. Raymond Grosset prend contact avec lui par courrier après avoir vu un de ses reportages publiés dans le journal $J$ et lui propose de «placer » certaines de ses photographies à l'étranger. Il lui précise les conditions de rétribution en cas de vente, à savoir un reversement de $50 \%$ des droits de reproduction perçus par l'agence. André Gamet rajoute que les termes acceptés oralement n'ont jamais fait l'objet d'un contrat mais la relation de confiance était telle que cet accord n'a connu aucun accroc. Rapho, une agence parisienne, film documentaire réalisé par Lily et Jean-Pierre Franey, co-produit par Regards productions multimédia et Demain TV, 2017 - 58'. 


\section{RÉSUMÉS}

Le fonctionnement des agences photographiques, notamment leur fonctionnement administratif et commercial, mais aussi les rapports entre les photographes et leurs agences restent pour beaucoup de responsables d'institutions en charge de la conservation de fonds photographiques provenant d'agences, de groupes de presse, de journaux ou revues, très largement méconnus voire étrangers. Cette difficulté à cerner les enjeux de production des photographies freine la mise en œuvre d'un plan de traitement et d'une valorisation adaptés à la spécificité de ces ensembles complexes. A l'occasion des journées d'études consacrées aux archives photographiques de presse organisées par les Archives nationales et la Mission de la photographie du ministère de la Culture en novembre 2015, il est apparu important de pouvoir préciser les mécanismes de production et de diffusion des reportages photographiques réalisés pour le compte d'agences, de qualifier les liens, en particulier les liens contractuels, qui unissent les photographes à leurs agences, d'appréhender les modalités de cession des photographies dans le cadre de leur activité commerciale. Très vite, il faut se rendre à l'évidence, ce souhait relève de la gageure. Chaque agence met en place ses processus de fonctionnement, de diffusion, ses règles de cession. Difficile voire impossible, dès lors, de généraliser. Aussi avons-nous voulu mieux comprendre une collaboration mythique entre un photographe et une agence en choisissant celle qui unissait Robert Doisneau à l'agence Rapho. Robert Doisneau est en effet l'homme d'une seule agence, Rapho, à laquelle il restera fidèle des premières photos publiées juste avant la guerre aux dernières, réalisées peu avant sa disparition en 1994. Ce long compagnonnage professionnel est d'abord dû à l'amitié et à la connivence qui lient le photographe au directeur de l'agence, Raymond Grosset et à ses collègues photographes Ergy Landau, Ylla, Emile Savitry, Willy Ronis, Sabine Weiss, Édouard Boubat... Qui mieux que Francine Deroudille, fille de Robert Doisneau qui fut aussi pendant vingt ans rédactrice à l'agence Rapho, pour rendre vie à cette collaboration ? Son point de vue à la fois d'ayant-droit mais aussi de salariée dans l'agence de son père, et donc sa connaissance des rouages et des pratiques de Rapho, sont autant de précieux atouts pour en appréhender l'organisation comme les principes de diffusion des tirages.

For the people in archive institutions who are responsible for the conservation of photo collections coming from photo agencies, press groups, newspapers or magazines, the way photographic agencies operated is by no means clearly understood, in particular the administrative and commercial activities of these agencies and the nature of their relations with their photographers. The difficulties in appreciating the issues involved in the production of photographic images often act as an obstacle to developing appropriate projects for treating and exploiting these complex documentary collections when they become archives. In November 2015, the French National Archives and the Photography Mission at the Ministry of Culture organised a study day devoted to press photo archives. The exchanges made it clear that better understanding was needed of the mechanisms of producing and diffusing photo reportages carried out for photo agencies, and, in particular, of the nature of the contractual links between the photographers and their agencies, and how the photographers might transfer their rights to the agency for the it's commercial activities. But it was soon apparent that this ambition was a difficult one to accomplish: each agency had its own way of operating and its own rules for diffusing pictures and transferring rights. It is consequently difficult, if not impossible to 
generalise. This is why we prefer here to look at the mythical collaboration between a particular photographer and his agency, Robert Doisneau and Rapho. As a photographer, Doisneau remained faithful to this one agency from the moment of his first photos published as an independent photographer just before the Second World War, up to his last works, taken shortly before his death in 1994. This long professional partnership is largely due to the friendship and understanding between the photographer and the agency's director, Raymond Grosset, and his photographer colleagues, Ergy Landau, Ylla, Emile Savitry, Willy Ronis, Sabine Weiss, Édouard Boubat... Who better than Francine Deroudille, Doisneau's daughter who was also an editorial secretary at the Rapho agency for more than twenty years, could explain this collaboration to us? Her point of view, both as a copyright beneficiary and as a member of the staff at her father's agency, her understanding, consequently, of how Rapho worked, are precious assets in appreciating the agency's operation and the way it diffused its photos.

\section{INDEX}

Keywords : press agency, Rapho, Magnum, Henri Cartier-Bresson, Robert Doisneau, copyright, Alliance Photo, Raymond Grosset, Charles Rado, Ergy Landau, working print, photographic archives

Mots-clés : agence de presse, Rapho, Magnum, Henri Cartier-Bresson, Robert Doisneau, droit d'auteur, Alliance Photo, Raymond Grosset, Charles Rado, Ergy Landau, tirages de travail, archives photographiques

\section{AUTEUR}

\section{INTERVIEW DE FRANCINE DEROUDILLE PAR ISABELLE-CÉCILE LE MÉE}

Chargée de mission photographie, direction générale des Patrimoines, ministère de la Culture isabelle.lemee@culture.gouv.fr 